\title{
THE USE OF FIBRIN AND POLY(LACTIC-CO-GLYCOLIC ACID) HYBRID SCAFFOLD FOR ARTICULAR CARTILAGE TISSUE ENGINEERING: AN IN VIVO ANALYSIS
}

\author{
S. Munirah ${ }^{1,2}$, S.H. Kim³ , B.H.I. Ruszymah ${ }^{1,2}$, G. Khang ${ }^{3 *}$ \\ ${ }^{1}$ Department of Physiology, Faculty of Medicine, Universiti Kebangsaan Malaysia, Jalan Raja Muda Abdul Aziz, \\ 50300 Kuala Lumpur, Malaysia \\ ${ }^{2}$ Tissue Engineering Laboratory, Universiti Kebangsaan Malaysia Hospital, $9^{\text {th }}$ floor, Clinical Block, Jalan Yaacob \\ Latif, 56000 Cheras, Kuala Lumpur, Malaysia \\ ${ }^{3}$ BK-21 Polymer BIN Fusion Research Team, Department of Polymer Science and Technology, Chonbuk National \\ University, 664-14, Dukjin, Jeonju, 561-756, South Korea
}

\begin{abstract}
Our preliminary results indicated that fibrin and poly(lacticco-glycolic acid) (PLGA) hybrid scaffold promoted early chondrogenesis of articular cartilage constructs in vitro. The aim of this study was to evaluate in vivo cartilaginous tissue formation by chondrocyte-seeded fibrin/PLGA hybrid scaffolds. PLGA scaffolds were soaked carefully, in chondrocyte-fibrin suspension, and polymerized by dropping thrombin-calcium chloride $\left(\mathrm{CaCl}_{2}\right)$ solution. PLGA-seeded chondrocytes were used as a control. Resulting constructs were implanted subcutaneously, at the dorsum of nude mice, for 4 weeks. Macroscopic observation, histological evaluation, gene expression and sulphated-glycosaminoglycan (sGAG) analyses were performed at each time point of 1,2 and 4 weeks postimplantation. Cartilaginous tissue formation in fibrin/PLGA hybrid construct was confirmed by the presence of lacunae and cartilage-isolated cells embedded within basophilic ground substance. Presence of proteoglycan and glycosaminoglycan (GAG) in fibrin/PLGA hybrid constructs was confirmed by positive Safranin O and Alcian Blue staining. Collagen type II exhibited intense immunopositivity at the pericellular matrices. Chondrogenic properties were further demonstrated by the expression of gene encoded cartilage-specific markers, collagen type II and aggrecan core protein. The sGAG production in fibrin/PLGA hybrid constructs was higher than in the PLGA group. In conclusion, fibrin/PLGA hybrid scaffold promotes cartilaginous tissue formation in vivo and may serve as a potential cell delivery vehicle and a structural basis for articular cartilage tissue-engineering.
\end{abstract}

Key Words: articular cartilage, chondrocytes, tissue engineering, scaffold, fibrin, poly(lactic-co-glycolic acid)

\footnotetext{
*Address for correspondence:

Gilson Khang

Biomaterials/Tissue Engineering Laboratory

Department of Polymer Science and Technology

Chonbuk National University

664-14, Dukjin, Jeonju, 561-756, South Korea

Tel: +82-63-270-2336
}

Fax: +82-63-270-2341

Email: gskhang@chonbuk.ac.kr, gskhang@krict.re.kr
Autologous chondrocyte implantation (ACI) has emerged as a successful viable alternative treatment in orthopaedics for arthroscopic intervention or joint replacement surgery (Brittberg et al., 1994; Mandelbaum et al., 2007; Peterson et al., 2003). Recently, further development of original ACI has led to the invention of in vitro three-dimensional (3D) cartilage implants that enabled easier surgical handling and facilitated cartilaginous tissue formation (Munirah et al., 2005; Munirah et al., 2007; Willers et al., 2005). This was supported by numerous investigations of the potential of full-thickness cartilage restoration, using $3 \mathrm{D}$ implants, in various animal models such as rabbit (Ito et al., 2005; Shao et al., 2006), goat (Niederauer et al., 2000), dog (Breinan et al., 2001) and sheep (Munirah et al., 2007). Since most studies reported implantation by means of arthrotomy, future improvement could be achieved by shifting this open surgery procedure to arthroscopy during the implantation of tissue-engineered construct.

We have successfully performed an autologous 'chondrocyte-fibrin' construct (ACFC) implantation to restore osteochondral defects in a sheep model (Munirah et al., 2007). Although the $3 \mathrm{D}$ nature of ACFC has allowed easier surgical handling and produced good results, at 12 weeks post-transplantation, the implantation was still carried out by means of open surgery. Besides, since ACFC was too soft to stay in the defect independently, periosteum - which was reported to induce formation of hypertrophic tissue (Peterson et al., 2000) - was used to secure ACFC into the osteochondral defect. Another trial on the restoration of osteochondral defects in patellar groove of rabbits, utilising the press-fit method and fibrin sealant, conducted by Uematsu et al. (2005), demonstrated that in some implants, consisting of PLGA-seeded cells, it had extruded from the defect. Therefore, in a search for ways to apply minimally invasive surgery for cartilage restoration, basic research is still necessary to develop its full potential. We believe a better outcome will be obtained by improving the scaffold materials of tissue-engineered constructs rather than by using currently available technique.

Hybridisation of various synthetically derived and naturally derived biodegradable polymers has been widely used for scaffold tissue engineering (Fan et al., 2006; Khang et al., 2003; Kim et al., 2006; Kim et al., 2007; Ko et al., 2007). Advantages of synthetically derived 
biodegradable polymers include controllable degradation rate, high reproducibility and ease of fabrication into specific shapes. Naturally derived biodegradable polymers usually mimick key elements of normal tissue (Khang et al., 2004; Park et al., 2005). Poly(lactic-co-glycolic acid) (PLGA) is bioabsorbable and biocompatible material, making it a promising material in the context of regenerative medicine. Numerous attempts have been made for successful tissue reconstruction using PLGAbased scaffold, either by PLGA itself (Park et al., 2005; Uematsu et al., 2005) or by incorporation of PLGA with natural polymers such as collagen (Chen et al., 2006a; Chen et al., 2006b), extracellular matrix scaffolds - namely small intestinal submucosa (Kim et al., 2006; Lee et al., 2004) and demineralised bone particles (Jang et al., 2005).

In the present study we aimed to evaluate in vivo formation of cartilaginous tissue in PLGA, utilizing a fibrin carrier matrix, after implantation in nude mice. Our preliminary results indicated that fibrin was an ideal cell transplantation matrix and enhanced in vitro chondrogenesis of rabbit articular chondrocytes (Munirah et al., 2008a) and intervertebral disc cells (Munirah et al., 2008b) in PLGA scaffolds. Fibrin, clinically applied as fibrin glue, is a natural polymer that forms during blood coagulation. Fibrin has been widely used for cartilage reconstruction purposes (Munirah et al., 2007; Munirah et al., 2006). Recently, Endres et al. (2007) showed that the 3D arrangement of human articular chondrocytes in human fibrin glue and resorbable polyglycolic acid (PGA) scaffolds, cultured in the presence of human serum, is an excellent system for the maturation of cartilage grafts in articular cartilage regeneration. Several previous studies also demonstrated that the fibrin immobilisation techniques promoted homogeneous cell distribution and bone formation, when using human periosteum-derived progenitor cells in PLGA (Zheng et al., 2006), PLGATCP composites (Arnold et al., 2002) and PLGApolydioxanon fleeces (Perka et al., 2000).

This report describes the formation of in vitro and in vivo cartilaginous tissue in a fibrin/PLGA scaffold. The results that demonstrated the feasibility of fibrin/PLGA as an ideal cell transplantation matrix for chondrogenesis and a structural basis for tissue-engineered articular cartilage are presented.

\section{Materials and Methods}

\section{Harvest of cartilage, chondrocytes isolation and monolayer culture expansion}

Articular cartilage was aseptically dissected from the femoral condyles and patellae of 6 weeks-old New Zealand White rabbits $(n=6)$. Each sample was placed in normal saline and processed within 6 to 12 hours post-surgery. Cartilage was washed with phosphate buffered saline (PBS $1 \mathrm{X}, \mathrm{pH}$ 7.2) (Gibco, Grand Island, NY, USA) containing $100 \mu \mathrm{g} / \mathrm{ml}$ penicillin and $100 \mu \mathrm{g} / \mathrm{ml}$ streptomycin (Gibco). Sample was then minced into small fragments and digested with $0.6 \%$ collagenase A (Roche Applied Science, Mannheim, Germany) at $37^{\circ} \mathrm{C}$ for 6 hours, for chondrocyte isolation. The resulting cell suspension was centrifuged at 1,500rpm, $5 \mathrm{~min}, 37^{\circ} \mathrm{C}$ (BHG, Hermle Z $360 \mathrm{~K}$, Wehingen, Germany). The supernatant was decanted and the cell pellet was washed with PBS to remove the remaining enzyme. After final centrifugation, the cell pellet was resuspended in PBS, for total cell count with a haemocytometer (Hausser Scientific, Horsham, PA, USA). Cell viability was determined using the trypan blue dye exclusion test (Gibco). Harvested chondrocytes were then seeded in 6 well-plates (Falcon, Franklin Lakes, NJ), with the initial seeding of 5,000 cells $/ \mathrm{cm}^{2}$ in the primary passage (P0).

Chondrocytes were cultured in the mixture of equal volume of F12 nutrient mixture (F12) and Dulbecco's Modified Eagle Medium (DMEM) (Gibco), supplemented with $10 \%$ foetal bovine serum (FBS) (Gibco) with the presence of antibiotics and antimycotic (Gibco), $200 \mathrm{mM}$ L-glutamine (Gibco), $50 \mu \mathrm{g} / \mathrm{ml}$ of ascorbic acid (Sigma) and $15 \mathrm{mM} \quad N$-2-hydroxyethylpiperazine-N'-2ethanesulfonic acid (HEPES) buffer 1M (Gibco) - as previously described (Ruszymah et al., 2007a; Ruszymah et al., 2007b). All cultures were maintained in a 5\% $\mathrm{CO}_{2}$ incubator (Optima Model 560, Optima Inc, Tokyo, Japan) at $37^{\circ} \mathrm{C}$, with the medium changed every other day.

\section{Preparation of microporous 3D PLGA scaffolds}

PLGA copolymer (mole ratio 50:50, molecular weight 33,000 g/mole, Resomer RG $503 \mathrm{H}$ ) was purchased from Boehringer Ingelheim Pharma GmbH (Ingelheim, Germany). Micro-porous 3D PLGA scaffolds were fabricated by solvent casting/salt leaching technique using methylene chloride $\left(\mathrm{CH}_{2} \mathrm{Cl}_{2}\right)\left(\mathrm{JT}\right.$ Baker, Baker Analyzed ${ }^{\circledR}$ A.C.S reagent, Selangor, Malaysia), as previously described (Kim et al., 2006; Khang et al., 2003). In this method, sieved sodium chloride $(\mathrm{NaCl})$ particles (approximately $180 \mu \mathrm{m}$ ) were dispersed in a polymer/ solvent solution $(0.2 \% \mathrm{w} / \mathrm{v}$ concentration of PLGA in methylene chloride), which was then cast to make a cylindrical disk scaffold using silicone moulds $(7 \mathrm{~mm}$ in diameter and $3 \mathrm{~mm}$ in thickness). The salt particles were leached out by continuous soaking in deionised water for 48 hours. This selective dissolution produces highly porous polymer. The 3D PLGA scaffolds were freeze-dried for 24 hours in a freeze-dryer (IlShin Lab Co., Seoul, South Korea), to remove any remaining solvent. The average of pore diameter; $139.4 \mu \mathrm{m}$ and porosity: $85.2 \%$ was determined by the mercury intrusion porosimeter; an AutoPore II 9220 (Micromeritics Co., Norcross, GA, USA). PLGA scaffolds were sterilised with $70 \%$ ethanol and washed three times with PBS before use.

\section{Formation of in vitro constructs}

Manufactured scaffolds were assigned into two experimental groups - cultured chondrocytes were seeded into (1) PLGA scaffolds with fibrin (fibrin/PLGA) and (2) PLGA scaffolds without fibrin. Cultured chondrocytes from the primary passage (P0) were trypsinised and subcultured (P1) in $75 \mathrm{~cm}^{2}$ culture flasks (Falcon) at a density of 5,000cells $/ \mathrm{cm}^{2}$. After the cells reached confluency, the P1 cells were harvested, counted for total cell and viability. Approximately, one million cells per scaffold were 
incorporated and resuspended in the (1) commercially available fibrin glue kit from Greenplast ${ }^{\circledR}$ (Green Cross P. D. Company, Yongin, Korea) and (2) culture medium. Greenplast fibrin glue kit consists of four vials - vial (1) lyophilized fibrinogen, (2) aprotinin solution, (3) lyophilized thrombin and (4) calcium chloride $\left(\mathrm{CaCl}_{2}\right)$ solution. Greenplast fibrinogen $(65-115 \mathrm{mg} / \mathrm{ml})$ was diluted in culture medium and the thrombin concentration (400-600 units/ml) was adjusted, using calcium chloride solution, in vial 4 to obtain a final working solution containing approximately $30-60 \mathrm{mg} / \mathrm{ml}$ fibrinogen and 10 50 units/ml thrombin - as suggested by the manufacturer. PLGA scaffolds were soaked carefully in the chondrocytefibrin suspension and polymerized, by dropping thrombin$\mathrm{CaCl}_{2}$ solution on to the scaffolds. For control group, PLGA scaffolds were soaked in chondrocytes suspended in culture medium. All constructs were cultured for 3 weeks in vitro, prior to implantation in vivo. Cell viability was assessed by a modified 3-(4,5-dimethylthiazole-2-yl)-2,5diphenyltetrazolium-bromide (MTT, Sigma, St. Louis, MO, USA) method that measured the reduction of a tetrazolium component (MTT) into an insoluble formazan product, by the mitochondria of viable cells. At a defined day, all constructs were transferred into new plates with 1 $\mathrm{ml}$ of new medium. $100 \mu \mathrm{l}$ of MTT solution $(5 \mathrm{mg} / \mathrm{ml}$ stock in PBS) was added into each well for $4 \mathrm{hrs}$ incubation at $37^{\circ} \mathrm{C}$. Next, all constructs were transferred into another new plate and the crystals formed were dissolved by using $1 \mathrm{ml}$ dimethylsulfoxide (DMSO, Sigma) per scaffold. 100 $\mu \mathrm{l}$ of solubilised mixture was transferred into $96-$ well microtiter plates and then the absorption intensity was analyzed using an ELISA plate reader at $590 \mathrm{~nm}$ (E-max, Molecular Device, MDS Analytical Technologies, Sunnyvale, CA, USA). Data were expressed as mean \pm standard error of mean (SEM) of 6 samples $(n=6)$.

\section{Implantation of the constructs}

Athymic nude mice were anaesthetized upon surgery. Minor skin incision was made at the dorsum of nude mice. Four constructs were implanted into the unconnected subcutaneous pockets of each nude mouse. Eighteen mice were used in this experiment (total implants, 72: PLGA/ fibrin $=36$, PLGA only $=36$ ). The resulting in vivo constructs were harvested at 1,2 and 4 weeks postimplantation. At time of explantation, all constructs were dissected free from the nude mice, weighed and processed for histological analysis, gene expression study and sulphated glycosaminoglycan (sGAG) content.

\section{Macroscopic observation, histological evaluation and immunohistochemistry analysis}

Each construct was observed grossly at room temperature, without any fixation, and palpated with forceps clinically to assess mechanical rigidity. Samples were then prepared for histological analyses. After fixation with $10 \%$ formalin for 24 hours, specimens were embedded in paraffin blocks and sectioned with a Leica microtome. All sections were then deparaffinised, rehydrated and stained with Haematoxylin and Eosin (H\&E) to assess tissue morphology, Safranin O staining, to identify the presence of proteoglycan-rich matrix, and Alcian blue staining, to detect accumulation of GAG, were applied.

Immunohistochemistry analysis was performed in accordance to the manufacturer's protocol (UltraTek HRP Kit, Immunotech, Marseille, France). All slides were deparaffinised, rehydrated and pre-treated with $0.4 \%$ pepsin (v/v 0.01N Hydrochloric acid) (Sigma-Aldrich, Inc. USA) at room temperature for 30 minutes. Slides were washed with PBS (Gibco). To reduce non-specific background staining, due to endogenous peroxidase, slides were incubated with hydrogen peroxide for 10 minutes. All sections were then treated with peroxidase block (Super Block, UltraTek HRP Kit; ScyTek Laboratories, Logan, UT, USA) for 10 minutes prior to antibody incubation. Monoclonal mouse anti-rabbit collagen type II (Calbiochem ${ }^{\circledR}$ EMD Biosciences Inc., La Jolla) and monoclonal mouse anti-rabbit collagen type I (Sigma Aldrich) antibodies were diluted $(1: 1000)$ with antibody DILUENT (DAKO Cytomation) and were applied to the sections for 60 minutes. After washing with PBS, UltraTek anti-polyvalent biotinylated antibody (UltraTek HRP Kit) was applied to the slides for 10 minutes at room temperature. Slides were then incubated with streptavidinperoxidase (UltraTek HRP Kit) for 10 minutes. After washing with PBS, the signal was finally visualized as a brownish precipitate, using freshly prepared chromogen substrate 3-amino-9-ethylcarbazole (AEC) (UltraTek AEC Kit). Sections were counterstained with Mayer's Haematoxylin and mounted, using permanent aqueous mounting medium (SkyTek Laboratories).

\section{Total RNA isolation, cDNA synthesis and conventional PCR}

Total RNA was extracted from in vitro and in vivo constructs to build up a profile of changes in gene expression. Approximately, $1.0 \times 10^{5}$ cells were homogenised using $1 \mathrm{ml}$ TRIzol reagent (Invitrogen, Carlsbad, CA). Cell lysate was centrifuged at 12,000 rpm, at $4^{\circ} \mathrm{C}$ for 5 minutes (Mega 17R, Hanil Science Industrial Co, Inchon, Korea). The supernatant was then transferred into a $15 \mathrm{ml}$ centrifugal tube (Falcon). Chloroform (SigmaAldrich) was added into the tube containing the supernatant and was then shaken vigorously for 10 seconds. After 10 minutes incubation at the room temperature, the mixture was then separated into an aqueous phase, containing RNA, an interphase and organic phase by centrifugation at 12,000 rpm for 15 minutes at $4^{\circ} \mathrm{C}$. The aqueous phase containing RNA was carefully transferred into a new $15 \mathrm{ml}$ centrifugal tube. Isopropanol (Sigma-Aldrich) was then mixed homogeneously into the tube, to precipitate the total RNA. $5 \mu \mathrm{l}$ of polyacryl carrier (Molecular Research Center, Cincinnati) was added to the mixture to facilitate RNA precipitation. The resulting total RNA was washed with $75 \%$ ethanol and air-dried. Yield and purity of the extracted RNA was determined spectrophotometrically (BioRad, Hercules, CA, USA).

Reverse transcription was carried out using Superscript ${ }^{\mathrm{TM}}$ II Reverse Transcriptase (Invitrogen, Carlsbad, CA, USA), according to the manufacturer's protocol. Total RNA (100-ng) was reverse transcribed in 


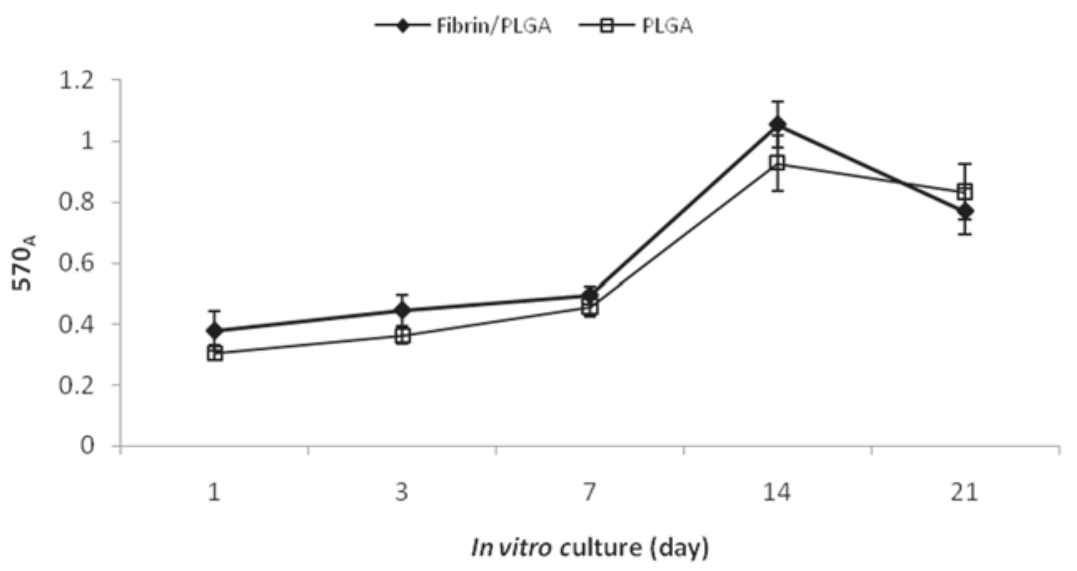

Figure 1. Fibrin/PLGA and PLGA group exhibited similar cell growth pattern in vitro (n=6). The MTT assay showed cell proliferation was gradually increased from day 1 until day 7, with fibrin/PLGA showing significantly higher cells proliferation activity $(p<0.05)$ compared to PLGA at day 3 . Next, cell proliferation activity in the fibrin/PLGA and PLGA was significantly increased by day 14. The proliferation activity then declined by day 21 in both groups.

a $31 \mu \mathrm{l}$ reaction volume containing oligo(dT)12-18 (500 $\mu \mathrm{g} / \mathrm{ml})$, dNTP mix $(10 \mathrm{mM})$, sterile distilled water, 5X First-strand buffer $(250 \mathrm{mM}$ Tris-HCl, $\mathrm{pH} 8.3$ at room temperature, $375 \mathrm{mM} \mathrm{KCl} ; 15 \mathrm{mM} \mathrm{MgCl}_{2}$ ), 0.1 M DTT, RNaseOUT $^{\mathrm{TM}}$ (40 units/ $\mu \mathrm{l}$ and Superscript ${ }^{\mathrm{TM}}$ II RT under the following conditions: $65^{\circ} \mathrm{C}$ for 5 minutes, $42^{\circ} \mathrm{C}$ for 2 minutes, $42{ }^{\circ} \mathrm{C}$ and $70{ }^{\circ} \mathrm{C}$ for 50 minutes and 15 minutes respectively). Polymerase chain reaction protocol was carried out in a $20 \mu \mathrm{l}$ reaction volume containing $10 \mu \mathrm{l}$ of $2 \mathrm{x}$ concentrated PCR master mix (Roche Applied Science, Germany), $3 \mu \mathrm{l}$ of reverse-transcribed reaction mixture, $10 \mu \mathrm{M}$ primers and sterile PCR water (Roche Applied Science) using the Takara Thermal Cycler (Takara Bio Inc. Japan). After initial denaturation of the templates at $94^{\circ} \mathrm{C}$, polymerase chain reaction was performed for 25 cycles at $94^{\circ} \mathrm{C}$ for 30 seconds, at $52^{\circ} \mathrm{C}$ for 30 seconds, at $72^{\circ} \mathrm{C}$ for 30 seconds and final extension at $72^{\circ} \mathrm{C}$ for 5 minutes. The PCR primers for detecting collagen type II, aggrecan core protein and collagen type I were designed from previous sequences as reported elsewhere (Ha et al. 2006; Yamazaki et al. 2002). Primer sequences were as follows: collagen type II: forward: 5'-gcacccatggacattggaggg-3' / reverse: 5'-atgttttaaaaaatacgaag-3' (Ha et al., 2006). Aggrecan core protein: forward: 5'-atcaacagagacctacgatgt-3'/ reverse: 5' gttagggtagaggtagaccgt-3' (Yamazaki et al., 2002). Collagen type I: forward: 5'-gatgcgttccagttcgagta-3' / reverse: 5'-ggtcttccggtggtcttgta-3' (Ha et al., 2006). The gene for rabbit $\beta$-actin was used as an endogenous control. The sequences for rabbit $\beta$-actin primer set (Yamazaki et al., 2002) were: forward: 5'-ccggcttcgcgggcgacg-3' / reverse: 5'-tcccggccagccaggtcc-3'. All primers were prepared by GenoTech. Corp. (Daejeon, Korea). $6 \mu$ of the amplified PCR products were separated by $1.5 \%$ agarose gel electrophoresis (SeaKem ${ }^{\circledR}$ LE Agarose, Cambrex Bio Science, Rockland, ME, USA), stained with SYBR $^{\circledR}$ Green Nucleic Acid Gel stain (Cambrex Bio Science) and visualised by UV transillumination, using a gel documentation system EDAS 290 Kodak (Viber Lourmat, Touray, France).

\section{Sulphated glycosaminoglycan (sGAG) production assay}

All samples were lyophilised and treated with papain digestion solution $(125 \mu \mathrm{g} / \mathrm{ml}$ of papain, $5 \mathrm{mM} \mathrm{L}$-cysteine, $100 \mathrm{mM} \mathrm{Na}_{2} \mathrm{HPO}_{4}, 5 \mathrm{mM}$ EDTA, pH 6.8) at $60^{\circ} \mathrm{C}$ for 16 hours. The sGAG contents were analyzed using a 1,9dimethylmethylene blue (DMMB) assay (Whitley et al., 1989). Each sample was mixed with the DMMB solution and the absorbance was measured at $590 \mathrm{~nm}$ wavelength. Total sGAG of each sample was extrapolated using a standard plot of shark chondroitin sulphate (Sigma Aldrich) in the range of $0-50 \mu \mathrm{g} / \mathrm{ml}$ and normalized by the dryweight of each sample $(n=6)$, as a relative sGAG content (\%). The dry-weight of each sample was determined after lyophilisation. Data was expressed as mean \pm standard error of the mean (SEM) of 6 samples $(n=6)$. Results were analyzed using Student's $t$-test and the difference are considered significance when $p<0.05$.

\section{Results}

\section{Cell proliferation and viability}

After cell seeding, cells adhered to the scaffolds, proliferated and produced cartilaginous matrices to fill void spaces in the scaffold. The fibrin/PLGA hybrid construct and PLGA (control) constructs exhibited similar cell growth pattern, as determined by means of MTT assay (Figure 1). From the chart, it can be seen that cell proliferation increased gradually from day-1 until day-7. The fibrin/PLGA hybrid construct showed a significantly higher cell viability and proliferation activity, compared to the PLGA at day-3. Next, by day-14, cell proliferation in the fibrin/PLGA and PLGA had increased significantly by $2.13 \mathrm{x}$ and $2.03 \mathrm{x}$, respectively. Cell proliferation then declined by day-21, in both groups. It has been indicated that the early stage of chondrogenesis involves the activity to establish cell-to-cell communication and cell-to-matrix interaction, with regards to new cartilaginous tissue 

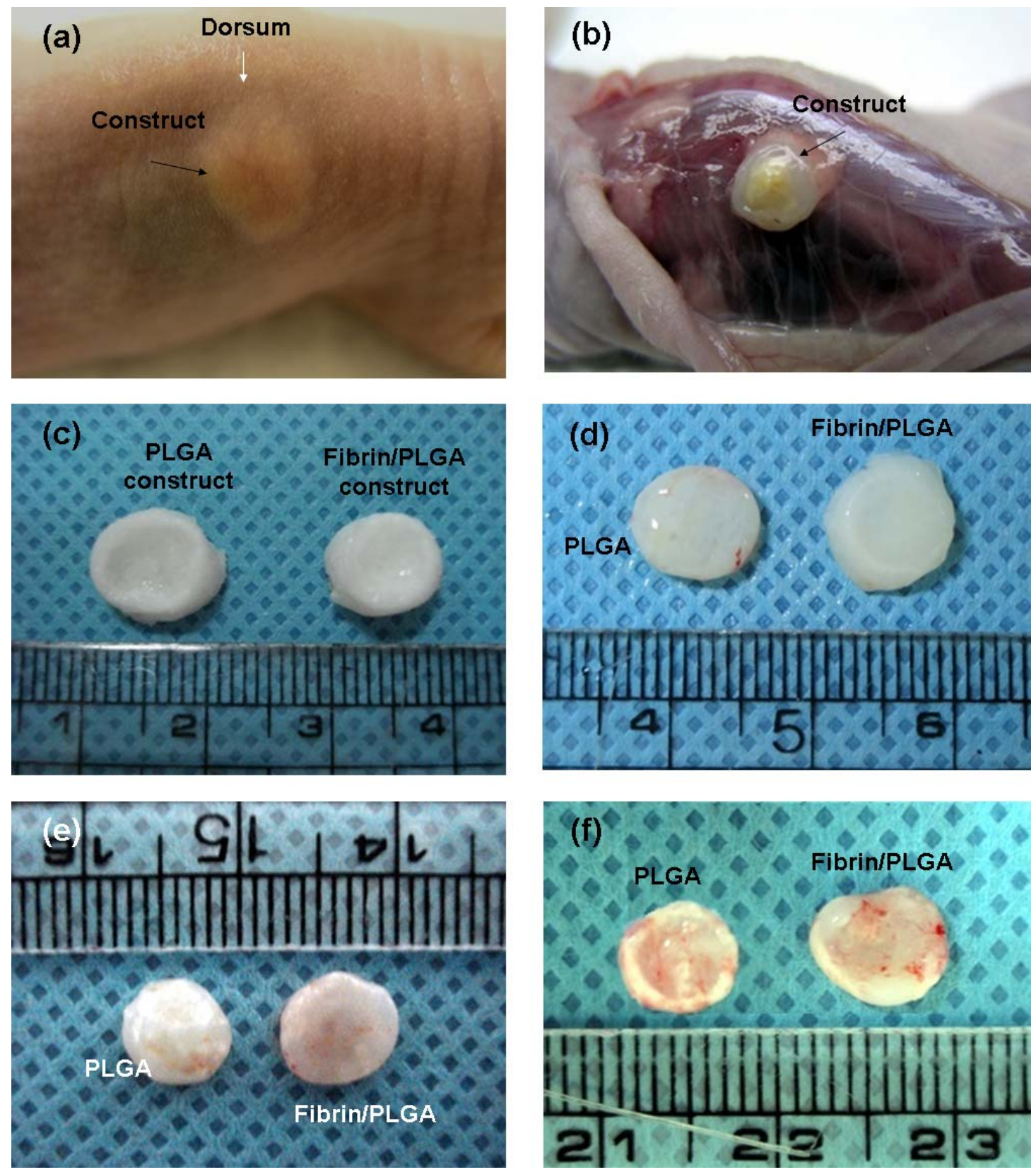

Figure 2. Figure 2a represents the subcutaneously implanted construct at the dorsum part of athymic nude mice. No evidence of superficial infection or fistula formation was demonstrated in the nude mice (Figure 2b). Figure 2c represents in vitro fibrin/PLGA and PLGA constructs before implantation. At time of explantation, gross examination showed no evidence of malignant invasion in any of the specimens. A thin vascularised capsule was observed surrounding the constructs. All fibrin/PLGA and PLGA constructs exhibited smooth and glistening appearance at each time point of 1 week (Figure 2d), 2 week (Figure 2e) and 4 weeks (Figure 2f).

formation, which causes cellular proliferation to become less active. This may be a possible explanation to a significant reduction of cell proliferation by $1.37 \mathrm{x}$ in the fibrin/PLGA construct after 21 days.

\section{Macroscopic observation of in vivo constructs}

After being cultured for 3 weeks in vitro, fibrin/PLGA hybrid and PLGA constructs were implanted subcutaneously at the dorsum of athymic nude mice (Figure 2a). After 1, 2 and 4 weeks implantation, no evidence of superficial infection or fistula formation was demonstrated in the nude mice. All constructs were easily dissected from the subcutaneous tissue (Figure 2b). After implantation, both fibrin/PLGA and PLGA constructs maintained their original rounded cylindrical shape, with a slight reduction in the size when compared to in vitro specimens. However, no significant variation was found between fibrin/PLGA and PLGA. Figure 2c represented in vitro fibrin/PLGA hybrid and PLGA constructs before implantation. All fibrin/PLGA hybrid and PLGA constructs exhibited smooth and glistening cartilage-like properties at each time point of 1 week (Figure 2d), 2 weeks (Figure 2e) and 4 weeks (Figure 2f) after implantation. Gross examination showed no evidence of malignant invasion in any of specimens. A thin vascularised capsule was observed surrounding the construct. Fibrin/PLGA hybrid and PLGA constructs preserved their original rounded shapes throughout the 4 week implantation. 

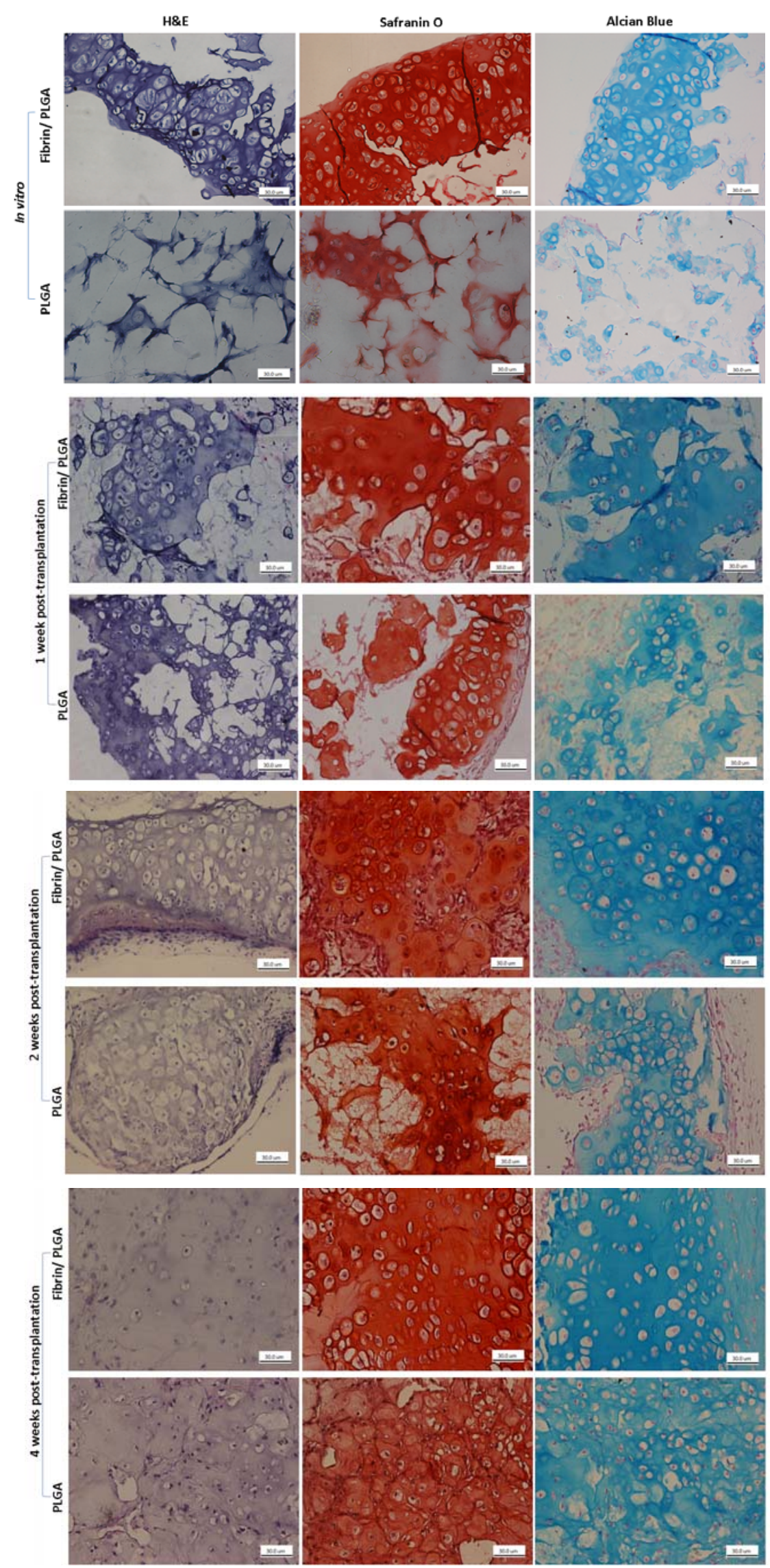

Figure 3. Before implantation (in vitro), a greater number of round morphological chondrocytes embedded in basophilic ECM were observed in fibrin/PLGA than in PLGA. The pericellular and inter-territorial matrix was strongly stained by Safranin O, to indicate presence of proteoglycan-rich matrix. The deposition of accumulated GAG was further demonstrated by positive Alcian blue staining. After 1, 2 and 4 weeks implantation, the in vivo fibrin/PLGA construct demonstrated superior cartilaginous histoarchitecture properties, when compared to PLGA, in terms of cells distribution, cells-matrix organization and overall ECM production. 

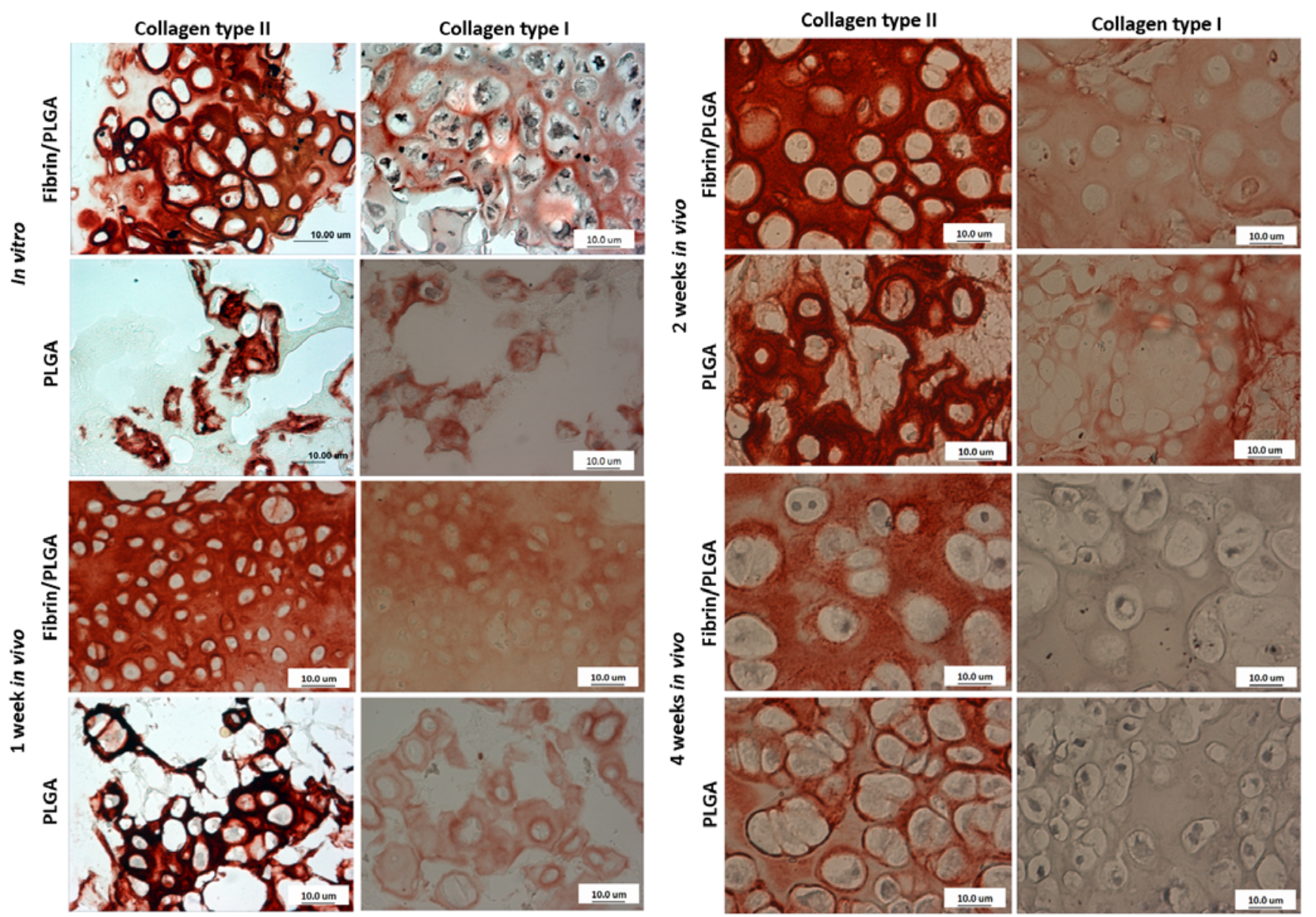

Figure 4. Collagen type II exhibited strong immunopositivity in the in vitro fibrin/PLGA constructs, mainly localised surrounding pericellular and inter-territorial matrix. After implantation, fibrin/PLGA showed more homogeneous distribution of collagen type II than PLGA. Collagen type I in fibrin/PLGA showed moderate immunopositivity throughout the ECM of specimens. Interestingly, after 4 weeks implantation, collagen type I showed either very weak or no expression in both fibrin/PLGA and PLGA.

Figure 5. Gel photos are representative of the mRNA expression analysis of in vitro and in vivo fibrin/PLGA $(\mathrm{n}=6)$ and PLGA constructs $(n=6)$ using RTPCR. The expression of genes encoded cartilage-specific markers; collagen type II and aggrecan core protein was gradually expressed in all constructs. Collagen type I was downregulated in fibrin/PLGA and PLGA group after implantation. $\beta$-actin gene was steadily expressed in all samples; to verify the analysis was reliable and successful.

Notes:

Aggrecan core protein:

M: $100 \mathrm{bp}$ marker

F/P: fibrin/PLGA

P: PLGA

IN VITRO:

1: week 1, 2: week 2, 3: week 3 IN VIVO:

1: week 1, 2: week 2, 4: week 4

\section{Collagen type I gene:} 312 bp 289 bp

Collagen type II: 394 bp
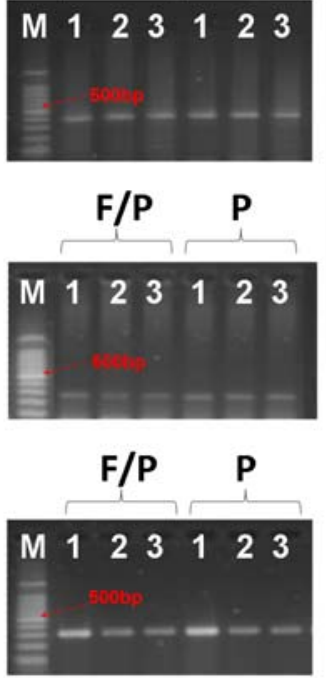

IN VITRO
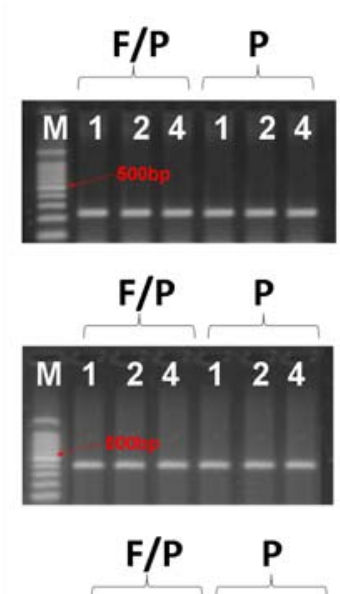

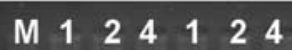

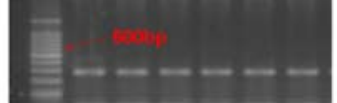

F/P P

M 12244124

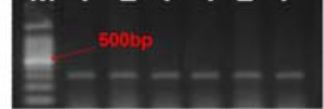

IN VIVO 


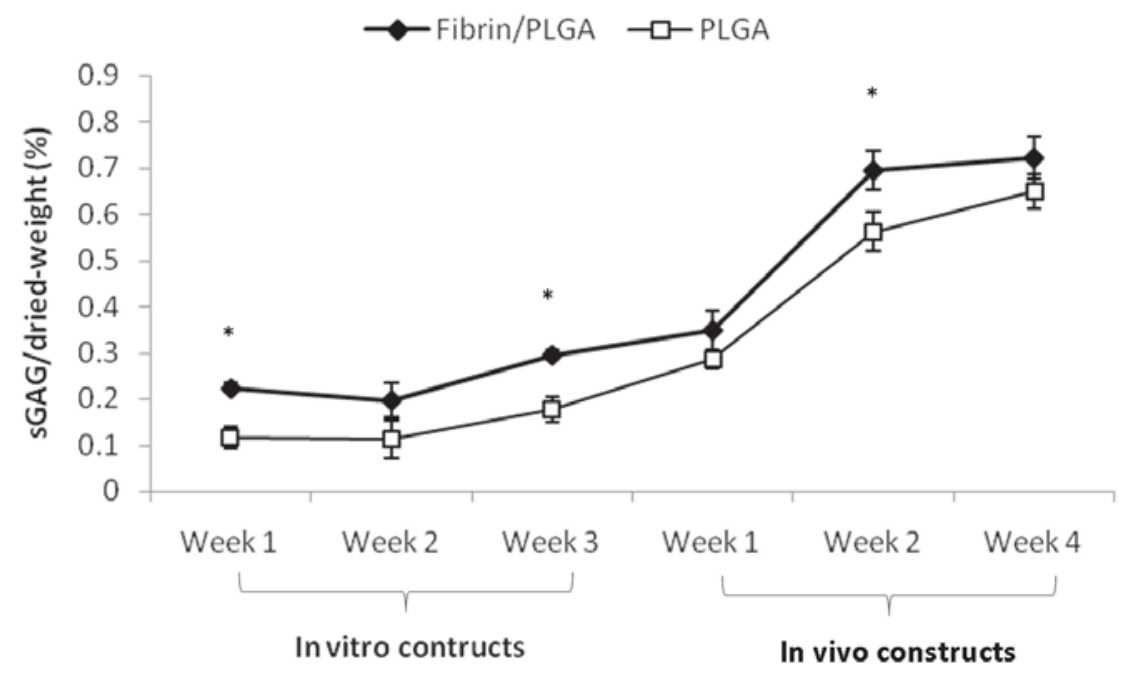

Figure 6. sGAG production in fibrin/PLGA was better than in PLGA at each time point before and after implantation $(\mathrm{n}=6)$. The relative sGAG contents $(\%)$ were significantly higher $(p<0.05)$ in fibrin/PLGA compared to PLGA at week 1 and week 3 in vitro and after 2-week implantation (*).

\section{Histological evaluation of in vitro and in vivo constructs}

The morphology of cells and distribution of cartilaginous ECM in the fibrin/PLGA hybrid and PLGA scaffolds were examined by means of histological staining; namely H\&E, Safranin O staining and Alcian blue staining (Figure 3). Before implantation, H\&E staining showed that chondrocyte clusters and ECM had filled void spaces of fibrin/PLGA hybrid scaffold. In vitro fibrin/PLGA constructs exhibited superior histo-architectural characteristics of cartilage-like tissue, when compared to the PLGA group. Closely-packed cartilage-isolated cells were homogeneously distributed in the basophilic ground substance. The pericellular and inter-territorial matrix was strongly stained by the characteristic red of Safranin O, to indicate presence of proteoglycan-rich matrix. Cartilaginous ECM deposition was further demonstrated by positive Alcian blue staining to confirm presence of accumulated GAG. Next, formation of cartilaginous tissue in fibrin/PLGA hybrid constructs was remarkably evidenced at each time point of 1,2 and 4 weeks after in vivo implantation. The difference between fibrin/PLGA hybrid construct and control group was clearly visible in term of overall cartilaginous tissue formation, cells organization and ECM distribution in the specimens. Fibrin/PLGA constructs exhibited good quality cartilagelike tissue, when compared to the control group.

\section{Immunohistochemistry analysis}

We analysed collagen type II and collagen type I immunolocalization in the fibrin/PLGA hybrid construct, and compared it with PLGA group at 1,2 and 4 weeks post-implantation (Figure 4). Collagen type II was detected in all specimens. Collagen type II exhibited strong immunopositivity at the specific region of in vitro fibrin/ PLGA hybrid construct, mainly localized at the pericellular and inter-territorial matrix. After in vivo implantation, fibrin/PLGA hybrid construct showed more homogenous collagen type II distribution than in the PLGA scaffold, at each time point. Analysis of collagen type I in fibrin/PLGA hybrid constructs showed moderate immunopositivity throughout ECM, at each time point of 1 and 2 weeks postimplantation. Interestingly, after 4 weeks implantation, $75 \%$ of implanted constructs showed no collagen I expression, whilst the rest showed very weak expression.

\section{Cartilage-specific phenotypic expression analysis}

When the mRNA expression of the fibrin/PLGA hybrid construct and PLGA group were compared, both showed comparable potential in sustaining the specific chondrogenic phenotype. The expression of gene encoded cartilage-specific markers, collagen type II and aggrecan core protein, was steadily observed in in vitro culture whereas collagen type I, the cartilage dedifferentiation marker, exhibited down-regulation pattern after 2 weeks in vitro. After implantation, collagen type II and aggrecan core protein mRNA expression was better than in vitro specimens. Collagen type I was detected in all in vivo specimens, but the expression was weaker than in the in vitro samples. The house-keeping gene, $\beta$-actin was steadily expressed in all specimens, to verify that the analysis was reliable and successful. Results were summarised in Figure 5.

\section{Sulphated glycosaminoglycan (sGAG) production assay}

Normalised by dry-weight of each sample $(n=6)$, the relative sGAG content (\%) was significantly higher $(p<0.05)$ in fibrin/PLGA hybrid constructs compared to control at 1 week and 3 week in vitro culture. With $0.223 \pm 0.010$ relative sGAG content, fibrin/PLGA hybrid constructs exhibited $1.92 \mathrm{x}$ higher sGAG production than the PLGA group; $0.116 \pm 0.025$ at week 1. At week-2 there was no significant difference between the two groups, with the relative sGAG content in fibrin/PLGA hybrid constructs being $0.197 \pm 0.037$ and PLGA group 0.113 \pm 0.042 . By week 3, fibrin/PLGA hybrid constructs exhibited $0.296 \pm 0.011$ relative sGAG content, which was $1.67 \mathrm{x}$ higher than the relative sGAG content in the PLGA group; $0.177 \pm 0.027$. After implantation, the relative sGAG 
content $(\%)$ in fibrin/PLGA hybrid construct was given by $0.349 \pm 0.043$ ( 1 week), $0.696 \pm 0.042$ ( 2 weeks) and $0.723 \pm 0.045$ (4 weeks). The relative sGAG content in fibrin/PLGA hybrid group increased significantly by 2 weeks post-implantation by $1.99 x$. While, in PLGA construct, the relative sGAG content $(\%)$ was $0.286 \pm 0.021$ ( 1 week), $0.562 \pm 0.042$ ( 2 weeks) and $0.650 \pm 0.038$ (4 weeks). Results were summarised in Figure 6.

\section{Discussion}

Tissue-engineered cartilage constructs should meet certain criteria in order to improve surgical handling and to withstand mechanical loading. Since synthetically derived biodegradable polymers can be moulded easily into the desired shapes with proper mechanical strength, they have been used as a skeleton for a mechanically weak natural polymer to withstand tissue collapse. The hybrid polymers used in this study are a Food and Drugs Administration (FDA) approved copolymer, lactic acid and glycolic acid, and the commercially available natural polymer fibrin from Greenplast ${ }^{\mathbb{R}}$. Fibrin was reported to be an ideal cell carrier (Eyrich et al., 2007), to provide homogenous cells distribution without significant cell loss, during cell seeding procedures (Zheng et al., 2006). In this study, we evaluated the formation of in vivo cartilaginous tissue using rabbit articular chondrocytes seeded in fibrin/PLGA hybrid scaffold at each time point of 1, 2, and 4 weeks postimplantation.

Our in vitro results indicated that chondrocytes-seeded in fibrin/PLGA secreted cartilage-specific ECM molecules and developed chondrocyte-chondrocyte interaction, to form clusters of various sizes, while preserving the original shapes of cells. Immobilization of cells in fibrin resulted in homogenous cell distribution in PLGA scaffolds. Moreover, the deposition of newly developed cartilaginous ECM in fibrin/PLGA was histologically superior to that of PLGA group. This phenomenon may be due to higher cell seeding efficiency and homogeneity in fibrin/PLGA than in PLGA. Homogenous distribution of cells was also observed during the osteogenesis of human periosteumderived progenitor cells in fibrin/PLGA (Zheng et al., 2006), while Lee et al. (2005) reported superior uniformity of chondrocytes, distributed in fibrin-filled polyurethane scaffold, when compared to polyurethane scaffold without fibrin.

After implantation, both fibrin/PLGA and PLGA constructs maintained their original rounded cylindrical shape, with a slight reduction in the size when compared to in vitro specimens. However, no significant variation was found between fibrin/PLGA and PLGA. All constructs showed cartilage-like properties with a white, smooth and glistening appearance, as reported elsewhere (Munirah et al., 2005, 2007). Fibrin/PLGA showed histologically mature chondrocytes and extensive development of cartilaginous ECM, indicated by presence of an abundant proteoglycan-rich matrix and accumulated GAG. Conversely, the PLGA group exhibited minimal cartilage histoarchitecture in vitro - indicated by few rounded cells in the void spaces of the scaffold. The entire PLGA scaffold was covered with newly developed cartilaginous ECM after only 4 weeks implantation. The incorporation of fibrin into the scaffold visibly facilitated formation of high quality cartilaginous tissue (Endres et al., 2007). Conversely, Jin et al. (2007) reported that in vitro differentiated human adipose-derived stem cells, seeded in PLGA, rapidly lost their chondrocytic phenotype and failed to produce cartilaginous tissue in vivo. Only fibrous tissues and angiogenesis invasions were observed after 4 weeks implantation.

Although there were remarkable histological differences between fibrin/PLGA and PLGA groups, there were no significant variations in the semi-quantitative gene expression for collagen type II, aggrecan core protein or collagen type I. Cartilage-specific chondrocyte phenotype was maintained in both groups. However, the expression of collagen type II and aggrecan core protein, improved after in vivo implantation, suggesting a mature cartilaginous phenotype. Interestingly, collagen type I expression demonstrated a down-regulation pattern after 2 and 3 weeks in vitro. However, the expression remained visible after 4 weeks implantation. On the contrary, immunohistochemistry against collagen type I denoted that $75 \%$ of implanted constructs showed no collagen I expression after 4 weeks implantation. This contradictory observation may have been due to the discrepancy between mRNA level and protein level analysis. Sasano et al. (1999; 2001) reported chondrocytes that rat tibial articular cartilage synthesised collagen type I and accumulated protein in ECM, during articular cartilage development. Absence of appropriate mechanical stimuli in the subcutaneous dorsum is believed to elicit some unfavourable effects on the maturation of neo-cartilage (Chen et al., 2004). Accordingly, the arrangement of articular chondrocytes in fibrin and PLGA scaffolds is an excellent system for maturation of cartilaginous tissues. Significant elevation of sGAG after implantation suggested progress towards development of cartilaginous tissue in fibrin/PLGA. If this result is applicable for future clinical use, a better outcome would be obtained than by using the currently available treatment methods.

\section{Conclusions}

Fibrin/PLGA scaffold promotes cellular proliferation and cartilaginous tissue formation of rabbit articular chondrocytes. This study suggests that fibrin/PLGA hybrid scaffold may serve as a potential cell delivery vehicle and a structural basis for tissue-engineered articular cartilage. Future study utilising autologous in vivo system in big animal model is necessary to validate the feasibility of fibrin/PLGA hybrid scaffold and cells composite for articular cartilage restoration.

\section{Acknowledgements}

This study was made possible by SCRC (SC3100) and KMOHW (0405-BO01-0204-0006). We thank Ms Youn 
Kyung Ko, Ms Hyun Jung Ha, Ms Jung Won So and the BK-21 Polymer BIN Fusion Research Team, Department of Polymer Science and Technology, Chonbuk National University, Korea who provided technical assistance for this study.

\section{References}

Arnold U, Lindenhayn K, Perka C (2002) In vitrocultivation of human periosteum derived cells in bioresorbable polymer-TCP-composites. Biomaterials 23: 2303-2310.

Breinan HA, Minas T, Hsu HP, Nehrer S, Shortkroft S, Spector M (2001) Autologous chondrocytes implantation in a canine model: Change in composition of reparative tissue with time. J Orthop Res 19: 482-492.

Brittberg M, Lindahl A, Nilsson A, Ohlsson C, Isaksson O, Peterson L (1994) Treatment of deep cartilage defects in the knee with autologous chondrocyte transplantation. N England J Med 331: 889-895.

Chen G, Sato T, Ushida T, Hirochika R, Ochiai N, Tateishi T (2004) Regeneration of cartilage tissue by combination of canine chondrocyte and a hybrid mesh scaffold. Mater Sci Eng C 24: 373-378.

Chen G, Sato T, Tanaka J, Tateishi T (2006a) Preparation of a biphasic scaffold for osteochondral tissue engineering. Mater Sci Eng C 26: 118-123.

Chen G, Tanaka J, Tateishi T (2006b) Osteochondral tissue engineering using a PLGA-collagen hybrid mesh. Mater Sc Eng C 26: 124-129.

Endres M. Neumann K, Schroder SEA, Vetterlein S, Morawietz L, Ringe J, Sittinger M, Kaps C (2007) Human polymer-based cartilage grafts for the regeneration of articular cartilage defects. Tissue and Cell 39: 293-301.

Eyrich D, Brandl F, Appel B, Wiese H, Maier G, Wenzel M, Staudenmaier R, Goepferich A, Blunk T (2007) Longterm stable fibrin gels for cartilage engineering. Biomaterials 28: 55-65.

Fan H, Hu Y, Zhang C, Li X, Lv R, Qin L, Zhu R (2006) Cartilage regeneration using mesenchymal stem cells and a PLGA-gelatin/chondroitin/ hyaluronate hybrid scaffold. Biomaterials 27: 4573-4580.

Ha HJ, Kim SH, Yoon SJ, Ko YK, Lee EK, Son Y, Kim MS, Rhee JM, Khang G, Lee BH (2006) Characterization of annulus fibrosus and nucleus pulposus with sequential passage. Tissue Eng Regen Med 3: 416422 .

Ito Y, Ochi M, Adachi N, Sugawara K, Yanada S, Ikada Y, Ronakorn P (2005) Repair of osteochondral defect with tissue engineered chondral plug in rabbit model. Arthroscopy: J Arthroscopic Related Surgery 21: 11551163.

Jang JW, Park KS, Kim SH, Park CS, Kim MS, Han CW, Rhee JM, Khang G, Lee HB (2005) Tissue engineered bone regeneration using DBP-loaded PLGA scaffold in rabbit model. Tissue Eng Regen Med 2: 34-40.

Jin XB, Sun YS, Zhang K, Wang J, Shi TP, Ju XD, Lou SQ (2007) Ectopic neocartilage formation from predifferentiated human adipose derived stem cells induced by adenoviral-mediated transfer of hTGF beta2. Biomaterials 28: 2994-3003.

Khang G, Lee SJ, Han CW, Rhee JM, Lee HB (2003) Preparation and characterization of natural/synthetic hybrid scaffolds. Adv Exp Med Biol 657: 235-245.

Khang G, Kim MS, Cho SH, Lee I, Rhee JM, Lee HB (2004) Natural scaffolds biomaterials for tissue regeneration. Tissue Eng Regen Med 1: 9-20.

Kim SH, Yoon SJ, Choi B, Ha HJ, Rhee JM, Kim MS, Yang YS, Lee HB, Khang G (2006) Evaluation of various types of scaffold for tissue engineered intervertebral disc. Adv Exp Med Bio 585: 169-181.

Kim SH, Ha HJ, Ko YK, Yoon SJ, Rhee JM, Kim MS, Lee HB, Khang G (2007) Correlation of proliferation, morphology and biological responses of fibroblasts on LDPE with different surface wettability. J Biomater Sci, Polym Ed 18: 609-622.

Ko YK, Kim SH, Ha HJ, Yoon SJ, Rhee JM, Kim MS, Lee HB, Khang G (2007) Hybrid type of tissue-engineered biodisc using annulus fibrosus seeded PLGA scaffold and nucleus pulposus seeded MPEG-PCL hydrogel: Preliminary Study. Key Eng Mater 342-343: 173-176.

Lee CR, Grad S, Gorna K, Gogolewski S, Goessl A, Alini M (2005) Fibrin-Polyurethane composites for articular cartilage tissue engineering: A preliminary analysis. Tissue Eng 11: 1562-1573.

Lee SJ, Lee I, Lee YM, Lee HB, Khang G (2004) Macroporous biodegradable natural/synthetic hybrid scaffolds as small intestine mucosa impregnated poly(lactide-Co-glycolide) for tissue engineered bone. J Biomater Sci, Polym Ed 15: 1003-1017.

Mandelbaum B, Browne JE, Fu F, Micheli LJ, Moseley JB, Erggelet C, Anderson AF (2007) Treatment outcomes of autologous chondrocyte implantation for full-thickness articular cartilage defects of the trochlea. Am J Sports Med 35: 915-921.

Munirah S, Aminuddin BS, Samsuddin OC, Chua KH, Fuzina NH, Ruszymah BHI (2005) The re-expression of collagen type II, aggrecan and sox 9 in tissue engineered human articular cartilage. Tissue Eng Regen Med 2: 347355.

Munirah S, Aminuddin BS, Chua KH, Samsudin OC, Badrul AHMY, Azmi B, Fuzina NH, Ruszymah BHI (2006) Tissue-engineered human articular cartilage demonstrates intense immunopositivity for collagen type II. J Biosciences 17: 9-18.

Munirah S, Samsudin OC, Chen HC, Sharifah Salmah SH, Aminuddin BS, Ruszymah BHI (2007) Articular cartilage restoration in load-bearing osteochondral defects by autologous chondrocytes-fibrin constructs implantation: An experimental study in sheep. J Bone Joint Surg (Br) 89-B: 1099-1109.

Munirah S, Kim SH, Ruszymah BHI, Khang G (2008a) Fibrin and poly(lactic-co-glycolic acid) hybrid scaffold promotes early chondrogenesis of articular chondrocytes: an in vitro study. J Orthop Surg Res, in press.

Munirah S, Yun SJ, Ko YK, Ha HJ, So JW, Kim SH, Ruszymah BHI, Khang G (2008b) Fibrin promotes cellular proliferation and matrix production of annulus fibrosus and nucleus pulposus cells cultured in three-dimensional 
poly(lactic-co-glycolic acid) scaffold. J Biomater Sci, Polym Ed, in press.

Niederauer GG, Silva MA, Leatherbury NC, Korvick DL, Harroff HH, Ehler WC, Dunn CJ, Kiewsetter K (2000) Evaluation of multiphase implants for repair of focal osteochondral defects in goats. Biomaterials 21: 25612574.

Park GE, Pattison MA, Park K, Webster TJ (2005) Accelerated chondrocyte functions on $\mathrm{NaOH}$-treated PLGA scaffolds. Biomaterials 26: 3075-3082.

Park SH, Park SR, Chung SI, Pai KS, Min BH (2005) Tissue-engineered cartilage using fibrin/hyaluronan composite gel and its in vivo implantation. Thoughts and Progress. Artif Organs 29: 838-845.

Perka C, Schultz O, Spitzer RS, Lindenhayn K, Burmester GR, Sittinger M (2000) Segmental bone repair by tissue-engineered periosteal cell transplants with bioresorbable fleece and fibrin scaffolds in rabbits. Biomaterials 21: 1145-1153.

Peterson L, Minas T, Brittberg M, Nilsson A, SjogrenJansson E, Lindahl A(2000) Two- to 9-Year Outcome After Autologous Chondrocyte Transplantation of the Knee. Clin Orthop Rel Res 374: 212-234.

Peterson L, Minas T, Brittberg M, Lindahl A (2003) Treatment of osteochondritis dissecans of the knee with autologous chondrocyte transplantation: results at two to ten years. J Bone Joint Surg Am 85 (Suppl 2): 17-24.

Ruszymah BHI, Chua KH, Farah Wahida I, Fuzina NH, Aminuddin BS (2007a) The Expansion Potential of Human Nasal Septum Chondrocytes for the Formation of Engineered Cartilage. Science Asia 33: 145-152.

Ruszymah BHI, Lokman BS, Asma A, Munirah S, Chua KH, Mazlyzam AL, Isa MR, Fuzina NH, Aminuddin BS (2007b) Pediatric auricular chondrocytes gene expression analysis in monolayer culture and engineered elastic cartilage. Int J Pediatr Otorhinolaryngol 71: 1225 1234.

Sasano Y, Furusawa M, Ohtani H, Mizoguchi I, Takahashi I, Kagayama M (1996) Chondrocytes synthesize type I collagen and accumulate the protein in the matrix during development of rat tibial articular cartilage. Anat Embryol (Berl) 194: 247-252.

Sasano Y, Takahashi I, Zhu JX, Ohtani H, Mizoguchi I, Takahashi I, Kagayama M (2001) Gene and protein expressions of type I collagen are regulated tissuespecifically in rat hyaline cartilages in vivo. Eur J Morphol 39: 149-154.

Shao XX, Hutmacher DW, Ho ST, Goh JCH, Lee EH (2006) Evaluation of a hybrid scaffold/cell construct repair of high load bearing osteochondral defects in rabbits. Biomaterials 27: 1071-1080.

Uematsu K, Hattori K, Ishimoto Y, Yamauchi J, Habata T, Takakura Y, Ohgushi H, Fukuchi T, Sato M (2005) Cartilage regeneration using mesenchymal stem cells and a three-dimensional poly-lactic-glycolic acid (PLGA) scaffold. Biomaterials 26: 4273-4279.

Whitley CB, Ridnour MD, Draper KA, Dutton CM, Neglia JP (1989) Diagnostic test for mucopolysaccharidosis I. Direct method for quantifying excessive urinary glycosaminoglycan excretion. Clin Chem 35: 374-379.
Willers C, Chen J, Wood D, Xu J, Zheng MH (2005) Autologous chondrocytes implantation with collagen bioscaffold for the treatment of osteochondral defects in rabbits. Tissue Eng 11: 1065-1076.

Yamazaki S, Banes AJ, Weinhold PS, Tsuzaki M, Kawakami M and Minchew JT (2002) Vibratory loading decreases extracellular matrix and matrix metalloproteinase gene expression in rabbit annulus cells' one hundred sixty years ago in spine. Spine J 2: 415-420.

Zheng YX, Ringe J, Liang Z, Loch A, Chen L, Sittinger M (2006) Osteogenic potential of human periosteumderived progenitor cells in PLGA scaffold using allogeneic serum. J Zhejiang Univ Science B 7: 817-824.

\section{Discussion with Reviewers}

S. Grad: The pore size of $90-180 \mu \mathrm{m}$ appears to be relatively small. A proper cell seeding procedure is therefore crucial to achieve a uniform cell distribution. It is unclear how the cells were seeded, since the descriptions in the abstract and methods section vary. Were different seeding methods evaluated? Also, a common observation in chondrocyte-seeded scaffolds is the development of a prominent layer rich in extracellular matrix at the edges of the constructs. Was this phenomenon also noticed in PLGA and/or fibrin/PLGA constructs?

Authors: The reviewer is correct. Initially, we seeded the cells directly onto scaffold but, as the reviewer mentioned above, a common observation in chondrocyte-seeded scaffolds is the development of a prominent layer rich in extracellular matrix at the edges of the constructs. Therefore, we tried to soak the scaffold carefully in cellsfibrin admixture and cell suspension in culture medium. To achieve a uniform cell distribution, we introduced some mechanical force, using a pipette, to aid cellular infiltration throughout the scaffold.

S. Grad: How do the amounts of sGAG/dry weight compare to values of native articular cartilage?

Authors: The aim of this study was not to regenerate a complete native cartilage tissue, that would be ideal for replacing articular cartilage loss, but more towards developing a cell delivery system for effective cartilaginous tissue formation from chondrocytes, as well as to demonstrate the applicability of fibrin-cells-PLGA complex. This is the reason why we only compared fibrin/ PLGA and PLGA.

D. Eglin: At the concentration of fibrinogen used, the viscosity of the solution is very high and by dropping the thrombin- $\mathrm{CaCl}_{2}$ on the top of the scaffold, without mixing, it is likely that the homogeneity of the gel in the PLGA scaffolds may not be optimal and the gel formation (e.g., cross-link density) may also be heterogeneous. (more cross-linking on the surface than in the bulk of the PLGA scaffolds. Did the authors check the fibrin gel homogeneity in the PLGA scaffolds? 
Authors: $65-115 \mathrm{mg} / \mathrm{ml}$ and $400-600$ units/ml is a working concentration of fibrinogen and thrombin recommended by the manufacturer for general procedure during surgery. However, since we were using cells, fibrinogen and the thrombin- $\mathrm{CaCl}_{2}$ solution concentrations had to be modified. The fibrinogen concentration was diluted to approximately $30-60 \mathrm{mg} / \mathrm{ml}$, with culture medium, and the thrombin solution was diluted to 10-50 units $/ \mathrm{ml}$, with $\mathrm{CaCl}_{2}$ solution, to achieve at least 15 to 30 sec clotting time - as suggested by the manufacturer. This has been included in the text. We did not check the fibrin gel homogeneity in PLGA, as such, but we did notice a homogenous formazan crystal distribution in the fibrin/ PLGA construct as well as the PLGA construct, during the MTT assay. We believe this phenomenon was very much associated with cells-fibrin homogeneity in PLGA. Furthermore, histological evaluation of in vitro constructs demonstrated a homogenous cells distribution in fibrin/ PLGA scaffold. 